\title{
Towards further experimental reproducibility: making a balance between conciseness, precision and comprehensiveness in scientific communication
}

Volume 3 Issue I - 2015

\section{Opinion}

Scientific research, usually starts with the researcher reading already published papers on the relevant topic, and ends by publishing results that other investigators will read and develop. Hence, publication in this field is of vital importance for continuous scientific discoveries, through building research new steps on results obtained previously. ${ }^{1}$ For that, it is crucial that each step of conducting experiments should be described comprehensively and precisely in an original paper. Unfortunately, this is not always the case, and I describe in this paper some illustrative instances and suggestions for improvement.

Reproducibility of a scientific experiment is highly important since it allows making sure that the results are reliable, and can be based on for further experiments. In order to achieve this endeavor, I put a spotlight on some examples. For instance, in cell culture experiments, almost all papers pass quickly over some steps description and leave the reader who wants to reconduct that experiment or a one based on it, unable to know necessary essential information, such as using a syringe or tips to discard the medium, the time between adding a reagent and the plate reading, how the initial aliquots of a drug were prepared and how long they were stored and at what temperature before being utilized. In addition, many papers do not mention the purity percentage of the drug tested and how long it was prepared with the culture medium before being given to the seeded cells and this makes the reader unclear about essential information. Another example is western blotting experimentsdescription; where many of the papers about this experiment steps neglect the clarification of the dilution method of the primary and secondary antibodies; being done in skimmed milk or a special solution for this process, and the specifications of all the reagents used in this experiment, such as those for preparing the loading buffer, etc. These steps are widely overlooked, but they are important to provide the reader with a precise description of the experiment, which is vital in scientific writing. This precision and comprehensiveness will allow the experimenter reading the paper able to reconduct it with certitude. Particularly, for instance, the purity of a compound; if not mentioned will make great troubles for the reader, since impurities may influence the therapeutic and the toxic effect of the tested drug.

In this regard, I suggest that the author of an original paper should make efforts to write detailed experiment process description. Herein, I further propose that scientific journals' editors have to insist on the point that the corresponding authors should reply to all inquiries of the readers about their papers. Companies providing chemical reagents, which after experimentation would be candidates in new drug discovery or tools to achieve finding out new therapies, are also invited to conduct more research to provide complete information about the

\author{
Besma Boubertakh, 1,2 \\ 'Key Laboratory of Animal Models and Human Disease, \\ Mechanisms of Chinese Academy of Sciences \& Yunnan Province, \\ Kunming Institute of Zoology, China \\ ${ }^{2}$ University of Chinese Academy of Sciences, China
}

Correspondence: Besma Boubertakh, Key Laboratory of Animal Models and Human Disease Mechanisms, Kunming Institute of Zoology, Chinese Academy of Sciences, No. 32 Jiaochang Donglu, Kunming 65223, China, Emailwww.boubertakhbesma@outlook.com

Received: October 02, 2015 | Published: October 05, 2015

reagents purchased, including the best solvent for the product, storage temperature after dissolving, and its stability requirements. I further suggest that the published paper should include photos of certain steps of the experiment to provide a clearer image about its process, which might help in a better reproducibility Following the tips suggested above, would hopefully guide towards better reproducibility of scientific experiments, which could make sturdier links between research laboratories all over the world and help to build new experimental designs based on reproducible experiments. Hence, the author of an original paper should make a certain balance, and not to consider that consciousness is more important than precision comprehensiveness in the communication of their research work. This could further enhance the fact that a scientist is presenting their results to the scientific community for objective discussion and for science development rather than covering a part of their work, since scientists should work for discovering science secrets and exchange ideas and findings with scientists all over the world in order to improve human health and the environment.

\section{Acknowledgments}

None.

\section{Conflicts of Interest}

The author declares that there is no conflict of interest.

\section{References}

1. Boubertakh B. Scientific Writing: A Push Forward Against Delaying. $J$ Psychol Clin Psychiatry. 2015;2(2):00062. 